\title{
Extensive psammomatous calcification of the uterus and cervix associated with a uterine serous carcinoma
}

\author{
R I Cameron, W G McCluggage
}

J Clin Pathol 2004;57:888-890. doi: 10.1136/icp.2004.017004

This report describes a uterine serous carcinoma with bilateral ovarian metastasis, which was associated with widespread extensive psammomatous calcification of the uterine leiomyomata, the myometrium, and the cervical stroma. These psammoma bodies were not associated with tumour or epithelial elements. This psammomatous calcification is rare, with no previous reports of similar cases. The presence of psammoma bodies is probably related to the serous carcinoma, raising the possibility that psammoma body formation in serous carcinomas is the result of a factor secreted locally by the tumour, rather than the widely held theory that their formation is secondary to necrosis, with subsequent dystrophic calcification within a papillary neoplasm.

$\mathrm{P}$ sammoma bodies are well circumscribed, laminated, calcified structures that can be seen in a range of conditions. They are especially common in the female genital tract, and are often associated with serous type neoplasms, which can be benign, borderline, or malignant. In some serous tumours, they are abundant and the term psammocarcinoma has been used for a highly differentiated serous carcinoma of the ovary or peritoneum associated with massive psammoma body formation. ${ }^{12}$ Psammoma bodies may also be associated with benign, non-neoplastic proliferations of serous type epithelium, termed endosalpingiosis. ${ }^{3}$ Occasionally, especially in peritoneal lined structures, psammoma bodies are found without an associated epithelial component, and it has been speculated that this represents "burnt out" or atrophic endosalpingiosis.

"We describe an unusual phenomenon of extensive psammoma body formation within uterine leiomyomas, the myometrium, and the cervical stroma"

Rarely, psammoma bodies are seen in the endometrium or cervical mucosa, sometimes in association with a serous proliferation elsewhere within the female genital tract. In this report, we describe an unusual phenomenon of extensive psammoma body formation within uterine leiomyomas, the myometrium, and the cervical stroma. These psammoma bodies were not associated with tumour or epithelial elements, but a small uterine serous carcinoma was present, which had spread to both ovaries. We speculate on the possible pathogenesis of psammoma body formation in this case.

\section{CASE REPORT}

A 56 year old woman presented with abdominal pain, nausea, and vomiting. Clinical examination revealed an acute abdomen and an emergency exploratory laparotomy

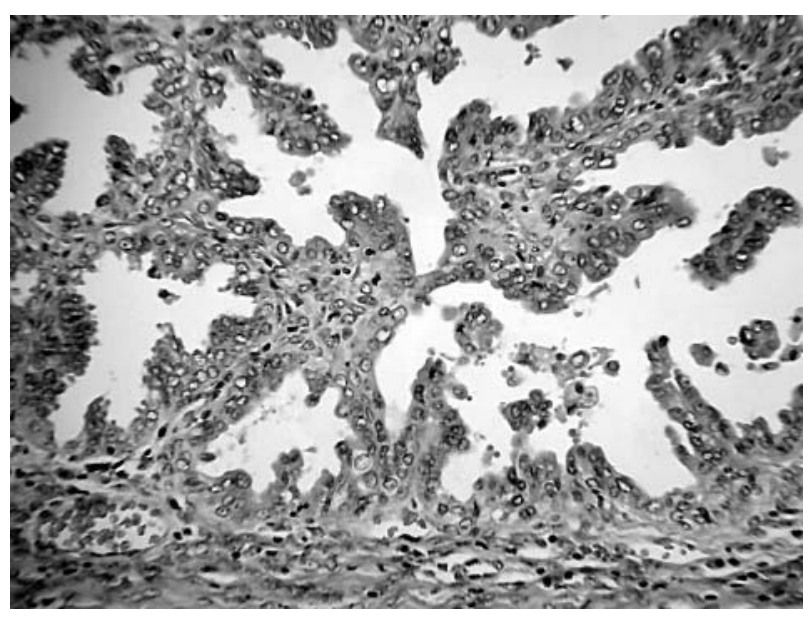

Figure 1 Poorly differentiated serous carcinoma involving the right ovary.

was performed. The uterus was found to be perforated and adherent to the descending colon. There was extensive ischaemic change of the colon and small intestine. A hysterectomy and bilateral salpingoophorectomy together with omentectomy, colectomy, and small intestinal resection was performed.

The uterus weighed $380 \mathrm{~g}$ and was perforated anteriorly. On sectioning, multiple fibroids were identified, the largest measuring $5 \mathrm{~cm}$ in diameter. No obvious endometrial or myometrial tumour was seen grossly. Both ovaries measured $4 \mathrm{~cm}$ in maximum diameter. Microscopically, the right ovary was entirely replaced by poorly differentiated serous carcinoma, with occasional psammoma bodies (fig 1). The left ovarian hilum contained similar serous carcinoma. A benign cystic teratoma (dermoid cyst) was also present within this ovary. There was involvement of the uterine serosa and the external surface of the cervix by serous carcinoma and, following the examination of multiple sections from the uterus, a small serous carcinoma with occasional psammoma bodies was seen within the endometrium (fig 2). No myometrial tumour was identified apart from the serosal tumour deposits. There was infiltration of the wall of the colon and small intestine by serous carcinoma and the colon and small intestine showed extensive ischaemic changes. There were no omental tumour deposits. The presence of benign uterine leiomyomata was confirmed. Immunohistochemistry revealed diffuse positivity of the tumour with an antibody to cytokeratin 7 (CK7) and negative staining for CK20. There was diffuse nuclear positivity for p53 but no staining for the Wilms's tumour 1 gene product (WT1).

Abbreviations: CK, cytokeratin; WT1, Wilms's tumour gene product 


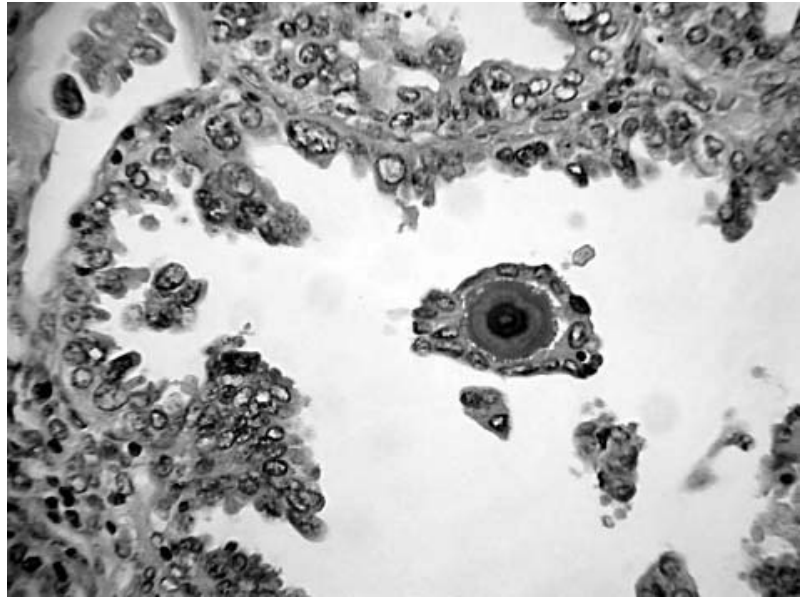

Figure 2 Serous carcinoma with occasional psammoma bodies involving the endometrium.

An additional unusual feature was the presence of numerous psammoma bodies throughout the leiomyomata (fig 3), the myometrium, and the cervical stroma, just beneath the surface epithelium (fig 4), and also deep within the stroma (fig 5). These formed aggregates and were associated with hyalinised fibrous tissue (fig 3). They were not associated with tumour or benign epithelial elements.

\section{DISCUSSION}

Psammoma bodies are characteristically associated with nonneoplastic serous proliferations and with serous neoplasms, which may be benign, borderline, or malignant. They are thought to arise secondary to necrosis, with subsequent dystrophic calcification of the tips of papillary structures. In our case, the presence of numerous psammoma bodies throughout the uterine leiomyomata, the myometrium, and the cervical stroma was extremely unusual and we are not aware of previous reports of similar cases. A serous carcinoma involved the endometrium and had metastasised to both ovaries, without evidence of myometrial involvement, except for tumour on the serosal surface of the uterus. The psammoma bodies in the myometrium and the cervix were not associated with epithelial elements, but we think that they are probably related in some way to the uterine serous

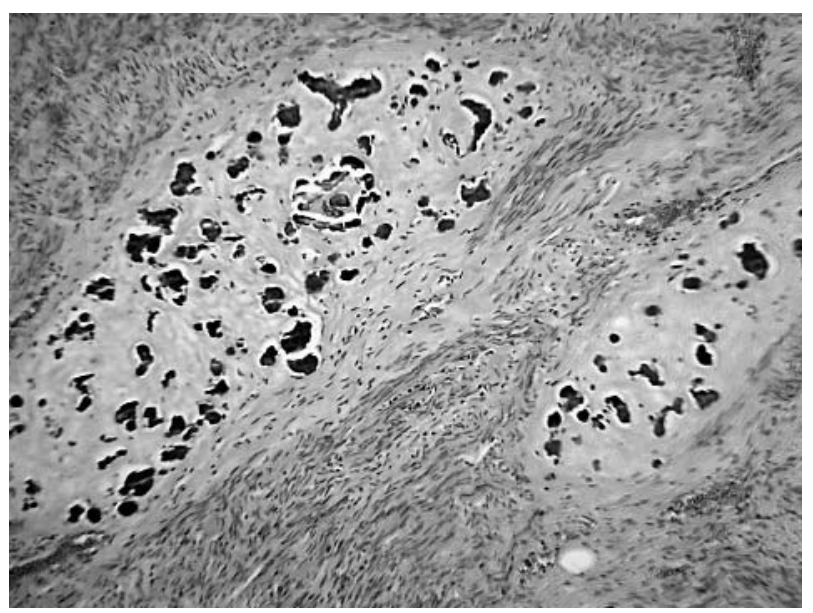

Figure 3 Aggregate of psammoma bodies associated with fibrous tissue involving a leiomyoma.

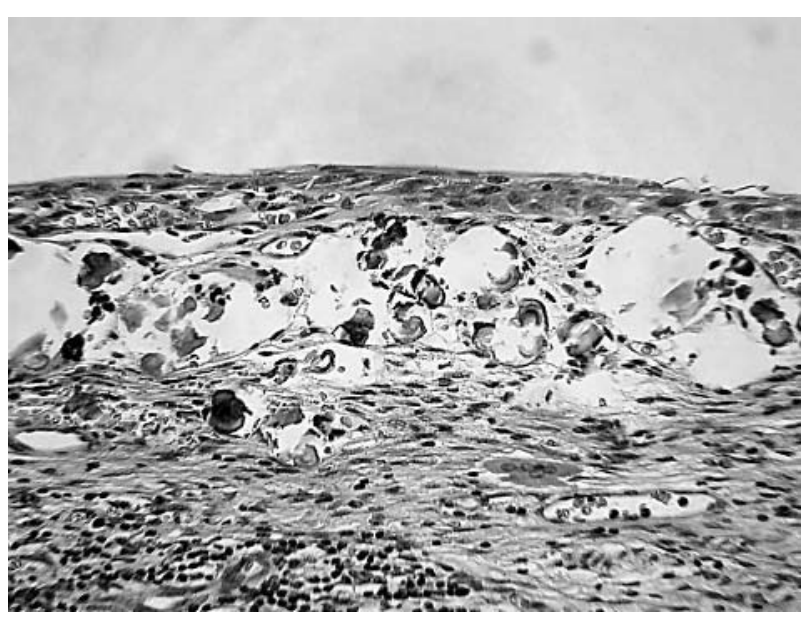

Figure 4 Psammoma bodies just beneath the surface epithelium of the cervix.

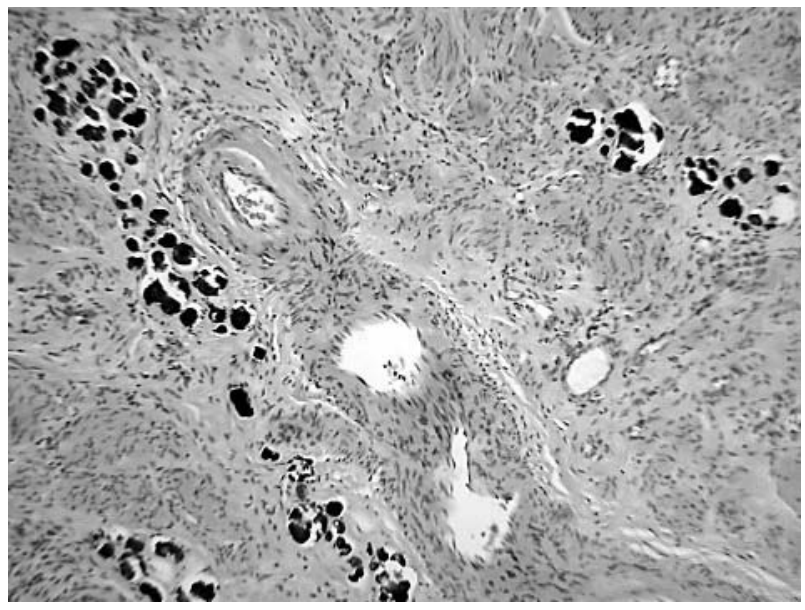

Figure 5 Psammoma bodies deep within cervical stroma.

carcinoma. One possibility is that they are the remnants of previous tumour within the myometrium and cervix, but that the tumour here has spontaneously regressed. However, we feel that this is extremely unlikely, and think that the psammomatous calcification is probably secondary to a local factor secreted by the tumour cells. It is not uncommon to find psammoma bodies without epithelial elements in the omentum or peritoneum in association with a serous tumour of the ovary. In this situation, it can also be postulated that the psammoma body formation is secondary to secretion of a factor by the tumour cells.

The presence of serous carcinoma within the endometrium and both ovaries raises questions regarding the origin of the tumour; that is, whether this represents a primary uterine serous carcinoma with bilateral ovarian metastasis or a primary ovarian carcinoma with endometrial metastasis. A further possibility is independent synchronous primaries within the endometrium and ovaries. We think that it is most likely that this represents a primary uterine neoplasm, but we cannot exclude the other possibilities. A pointer towards a uterine primary is that there was no staining of tumour cell nuclei with WT1. WTl positivity is usual in ovarian serous carcinomas but most uterine serous carcinomas are negative..$^{4-7}$ Moreover, there was no evidence of omental involvement by tumour, which is usual when a serous carcinoma spreads beyond the ovary. 


\section{Take home messages}

- We describe an unusual phenomenon of massive psammoma body formation, not associated with epithelial elements, throughout the uterine leiomyomata, the myometrium, and the cervical stroma

- This was associated with a uterine serous carcinoma that had metastasised to both ovaries

- This appears to be the first report of this phenomenon

- We speculate that psammoma body formation was secondary to a local factor secreted by the tumour

The uterus was found to be perforated at laparotomy. Spontaneous uterine perforation is rare and in this case was presumably secondary to the underlying carcinoma. However, it is possible that the numerous psammoma bodies throughout the myometrium contributed to the perforation. Uterine perforation may rarely occur as a complication of hysteroscopy and/or endometrial biopsy. However, there was no such history in our case.

"The presence of serous carcinoma within the endometrium and both ovaries raises questions regarding the origin of the tumour"

In addition to being associated with serous proliferations, psammoma bodies in the female genital tract can rarely be seen in other circumstances. Endometrial psammoma bodies may be seen in non-neoplastic conditions such as Asherman's syndrome, as a result of a reparative response, and they have been reported with longterm intrauterine contraceptive device usage, ${ }^{9}$ and in association with hormonal preparations. ${ }^{10}{ }^{11}$ Psammoma bodies may rarely be seen in uterine neoplasms other than serous carcinoma, such as endometrioid adenocarcinoma. ${ }^{12}$ Endometrial calcification associated with ossification may occasionally be encountered as a sequel to a spontaneous abortion, ${ }^{13}{ }^{14}$ which may have occurred years earlier, or may rarely be secondary to osseous metaplasia of the endometrial stroma. Endometrial ossification may result in infertility or repeated spontaneous abortions. ${ }^{15}{ }^{16}$ Rarely, endometrial psammoma bodies occur with no known predisposing cause. In such a situation, the question arises as to whether investigations should be performed to assess the entire female genital tract to exclude a serous proliferation. In one study of 11 women who were found to have psammoma bodies incidentally on endometrial biopsy, and who underwent further investigations, all cases were associated with benign findings, most often endometrial polyp. ${ }^{17}$

Psammoma bodies may rarely be seen in cervical smears, sometimes in association with the non-neoplastic conditions described..$^{10}{ }^{18}$ However, the presence of psammoma bodies in a cervical smear may also be a manifestation of a neoplastic lesion in the female genital tract. In particular, they have been associated with ovarian, fallopian tube, endometrial, and endocervical serous tumours. ${ }^{19-22}$ It is thought that in these cases psammoma bodies migrate through the fallopian tube and uterine cavity to present in cervical smears.

Myometrial calcification as a result of degenerative changes or following embolisation ${ }^{23}$ may occur in leiomyomas or in blood vessel walls in Monckeberg's medical calcific sclerosis. A case of massive myometrial necrosis and resultant dystrophic calcification secondary to shock has been reported in association with postpartum haemorrhage. ${ }^{24}$

In summary, we describe an unusual phenomenon of massive psammoma body formation, not associated with epithelial elements, throughout the uterine leiomyomata, the myometrium, and the cervical stroma. This was associated with a uterine serous carcinoma that had metastasised to both ovaries. In a search of the literature, we could find no previous reports of this phenomenon. We speculate that psammoma body formation in our case is secondary to a local factor secreted by the tumour.

\section{Authors' affiliations}

R I Cameron, W G McCluggage, Department of Pathology, Royal Group of Hospitals Trust, Belfast BT12 6BL, Northern Ireland

Correspondence to: Dr W G McCluggage, Department of Pathology, Royal Group of Hospitals Trust, Grosvenor Road, Belfast BT12 6BL, Northern Ireland; glenn.mccluggage@bll.n-i.nhs.uk

Accepted for publication 22 March 2004

\section{REFERENCES}

1 Gilks CB, Bell DA, Scully RE. Serous psammocarcinoma of the ovary and peritoneum. Int J Gynecol Pathol 1990;9:110-21.

2 Weir M, Bell DA, Young RH. Grade 1 peritoneal serous carcinomas: a report of 14 cases and comparison with 7 peritoneal serous psammocarcinomas and 19 peritoneal serous borderline tumors. Am J Surg Pathol 1998;22:849-62.

3 Zinsser KR, Wheeler JE. Endosalpingiosis in the omentum: a study of autopsy and surgical material. Am J Surg Pathol 1982;6:109-17.

4 Shimizu M, Toki T, Takagi $Y$, et al. Immunohistochemical detection of the Wilms' tumor gene (WT1) in epithelial ovarian tumors. Int J Gynecol Pathol 2000;19:158-63.

5 Al-Hussaini $M$, Stockman A, Foster $\mathrm{H}$, et al. WT-1 assists in distinguishing ovarian from uterine serous carcinoma and in distinguishing between serous and endometrioid ovarian carcinoma. Histopathology 2004;44:109-15.

6 Goldstein NS, Uzieblo A. WT-1 immunoreactivity in uterine papillary serous carcinomas is different from ovarian serous carcinomas. Am J Clin Pathol 2002; 117:541-5.

7 Hashi A, Yuminamochi T, Murata S, et al. Wilms' tumor gene immunoreactivity in primary serous carcinomas of the fallopian tube, ovary, endometrium and peritoneum. Int J Gynecol Pathol 2003;22:374-7.

8 Untawale VG, Gabriel JB, Chauhan PM. Calcific endometritis. Am J Obstet Gynecol 1982;144:482-3.

9 Gosden C, Ross A, Louden NB. Intrauterine deposition of calcium on copper bearing intrauterine contraceptive devices. BMJ 1977;1:202-6.

10 Valicenti JF, Priester SK. Psammoma bodies of benign endometrial origin in cervicovaginal cytology. Acta Cytol 1977;21:550-2.

11 Herbold DR, Magrane DM. Calcification of the benign endometrium. Arch Pathol Lab Med 1986;1 10:666-9.

12 Pakash V, Carcangiu ML. Endometrioid endometrial adenocarcinoma with psammoma bodies. Am J Surg Pathol 1997;21:399-406.

13 Ceccacci L, Clancy G. Endometrial ossification: report of an additional case. Am J Obstet Gynecol 1981;141:103-4.

14 Ganem KJ, Parsons L, Friedell GH. Endometrial ossification. Am J Obstet Gynecol 1962;83:1592-4.

15 Bahceci M, Demirel LC. Osseous metaplasia of the endometrium: a rare cause of infertility and its hysteroscopic management. Hum Reprod 1996;11:2537-9.

16 Degani S, Gonen R, de Vries K, et al. Endometrial ossification associated with repeated abortions. Acta Obstet Gynecol Scand 1983;62:281-2.

17 Fausett MB, Zah CM, Kendall BS, et al. The significance of psammoma bodies that are found incidentally during endometrial biopsy. Am J Obstet Gynecol 2002;186:180-3

18 Seguin RE, Ingram K. Cervicovaginal psammoma bodies in endosalpingiosis. A case report. J Reprod Med 2000;45:526-8.

19 Benson PA. Cytologic diagnosis in primary carcinoma of fallopian tube. Acta Cytol 1974; 18:429.

20 Parkash V, Chacho MS. Psammoma bodies in cervicovaginal smears: incidence and significance. Diagn Cytopathol 2002;26:81-6.

21 Kirkland N, Hardy N. Psammoma bodies found in cervicovaginal smears. Acta Cytol 1979;23:131-3.

22 Seltzer V, Spitzer M. Psammoma bodies in papillary adenocarcinoma of the endocervix. Int J Gynecol Pathol 1983;2:216-21.

23 McCluggage WG, Sloan JM, Traub Al. Postpartum diffuse calcification of the myometrium. Int J Gynecol Pathol 1996:1582-4.

24 McCluggage WG, Ellis PK, McClure N, et al. Pathologic features of uterine leiomyomas following uterine artery embolization. Int I Gynecol Pathol $2000 ; 19: 342-7$. 